\title{
Video-based Simulations: Considerations for Teaching Students with Developmental Disabilities
}

\author{
CHERYL A. WISSICK \\ The University of South Carolina \\ J. EMMETT GARDNER \\ The University of Oklahoma \\ JOHN LAANGONE \\ The University of Georgia
}

\begin{abstract}
The use of video-based multimedia simulations for teaching functional skills to persons with developmental disabilities remains an unexplored application of technology for this group. This article examines the historical literature in this area, and discusses future considerations, design issues, and implications of using multimedia simulations. Implementation issues are presented, and suggestions regarding design, development, and application of multimedia simulations are offered. Considerations address the importance of appropriate role modeling and the combination of video-based simulation and in vivo training to foster generalization and maintenance in the context of transition to the real world.
\end{abstract}

The results of research indicate that successful community-based living and employment for persons with developmental disabilities is linked to the functional skills and knowledge they possess relevant to their home, community, and employment situations (Cuvo \& Klatt, 1992; Wolfe, 1994). When these skills are taught in the context of community-based instruction, learning is more efficient and long term (Langone, 1990). Community-based instruction is a powerful approach for teaching functional living and vocational skills and has been shown to enhance students' learning of skills needed to become independent in their adult lives (McDonnell, Hardman, Hightower, Keifer-O'Donnell, \& Drew, 1993; Snell \& Browder, 1986).

One issue associated with the delivery of community-based instruction is that the logistics of getting students to training sites frequently enough for learning to occur can pose problems because of travel and scheduling con- 
flicts. Teachers faced with such logistical problems often resort to developing more traditional classroom-based activities. Traditional classroom-based activities (e.g., lecturing about appropriate shopping skills, modeling social skills necessary to get along with coworkers) often do not provide an accurate depiction of the outcomes that are desirable for the target learners and will not facilitate transfer of learned skills to natural environments (Stokes \& Osnes, 1989). Classroom-based simulations have been used with some success. However, efforts to reproduce accurate depictions of community sites can be time and cost intensive (Morrow \& Bates, 1987). What is needed is a set of instructional techniques that can provide learners with accurate depictions (e.g., compressed video, photographs) of the multiple exemplars located in community settings in an efficient manner.

Computer-based instructional applications can provide learners with almost limitless examples to support important concepts and skills. These technology solutions can also provide students with the ability to interact with the electronic environment, as opposed to being passive learners. Technology solutions related to computer-based instruction have offered many teachers the ability to provide daily skill practice for students with mild or learning disabilities (e.g., Lewis, 1993; Lindsey, 1999; and Male, 1997). As research in computer-based instruction has advanced, results indicate that instruction which incorporates a video-based component to teach concepts or skills creates a more realistic learning environment than the regular classroom (Cognition and Technology Group at Vanderbilt [CTGV], 1993a; 1994). The video provides a visual anchor for students to relate their learning to real-life situations. The authenticity of the video environment appears to create a classroom learning situation that is representative of the typical environment in which the learner will ultimately be performing the skills. Considering the potential of video-based computer instruction for providing more realistic simulated environments within the classroom, there is a paucity of research that examines the effectiveness of this technology for supplementing functional skills that are also taught concurrently in community environments.

The purposes of this article are to (a) present instructional techniques that can be applied to the design and development of video or computerbased activities, (b) review the use of computer-based and video-based instruction for students with developmental disabilities, and (c) discuss implications for the application of multimedia simulations. The major focus of this article is to review the research on the use of computer and video technology for students with developmental disabilities. This review also will provide implications for the possible effectiveness of instructional technology to teach community-based skills.

We recognize that in an ideal teaching situation, students with develop- 
mental disabilities should be taught in community settings as much as possible. However, due to the constraints mentioned earlier (e.g., transportation and scheduling), many students make infrequent visits into the community. The need for alternative methods would be greatest for those skills that present the practical problems of cost, safety, travel, and time and for those skills that have a high priority for promoting student independence. For teachers who are unable to provide sufficient community-based instruction because of logistical constraints, technology-based instructional tools are needed that present their students with relevant instructional contexts with interactive practice related to real-life activities. Such tools designed to augment instruction in natural settings may present an efficient set of strategies that can ultimately decrease the time involved to master skills once the students do interact in community environments.

\section{BEHAVIORAL INSTRUCTION AND TECHNOLOGY}

The extant literature on general case programming demonstrates that the use of behavioral strategies can be highly effective in teaching functional community-based skills (e.g., McDonnell et al., 1993). In addition, a technology-based instructional approach, "anchored instruction," has been successfully used to provide learners with rich video-based environments that serve to assist them in concept development, application of academic skills, and problem- solving behaviors (CTGV, 1993a). These approaches are highly compatible since the technology involved in providing students with video environments and examples of key exemplars can overcome the problem of spending large amounts of time in natural settings.

Research in the areas of community-based instruction and instructional technology can provide us with a knowledge base for creating effective instructional tools. Instructional technology research contributes information on how to create visual models and interactive environments. This research base and its link to creating effective simulations are examined in the next section.

\section{Effective Simulation Techniques}

The term "simulation" can be used to describe a wide variety of instructional activities, methods, or materials. Computer-based simulations have been used to represent the functioning of another system or event, real or fantasy, providing an experiential awareness of a process or event. For students with developmental disabilities, simulations must be age-appropriate and functional, providing a method for the students to achieve performance in natural settings (Horner, McDonnell, \& Bellamy, 1986). Therefore, the medium chosen for development of the simulation should approximate 
that natural environment and provide the teacher with a tool for increasing the experiences for interaction.

In order to develop effective instruction for students with developmental disabilities, teachers have to decide on which skills to train. Considerations for a community skill include the importance of the skill in fostering independence and transition. A number of publications provide readers with detailed information regarding the use of ecological inventories for identifying important skills for instruction (e.g., Snell \& Browder, 1986). Teachers may need to develop alternative activities or simulations for those skills that would be the most difficult to train in the community. For example, training the skills for grocery shopping, requiring trips to different stores, or street crossing in high-traffic areas, would be more difficult than training laundry skills if the facilities are available at the school.

Recognizing that simulation training should be used as an adjunct to, not a replacement for, community training, McDonnell \& Ferguson (1988) compared the effectiveness and efficiency of training in the natural environment to instruction in both the natural environment and the classroom. Instruction in the natural environment was more efficient (as measured as the number of trials to criterion and the cost of the instruction) than the instruction in both environments, but the strategy using both environments promoted greater generalized responding.

Factors concerning generalization need to be considered when evaluating or designing the use of a simulation. Generalization should be assessed (a) in the community setting and not just the simulated setting and (b) in several non-trained natural settings. Without these generalization components, the effectiveness of the use of a simulation to train the performance of behaviors across the full range of situations cannot be measured (McDonnell \& Horner, 1985).

The results of research indicate that in order for students to perform skills and generalize those skills in a variety of settings, they had to be taught in several natural settings (McDonnell, Horner, \& Williams, 1984). Given the problems of training in the community and the additional problems of providing multiple experiences in the natural setting, researchers explored the use of simulations as an alternative for providing a range of experiences. Neef, Lensbower, Hockersmith, DePalma, and Gray (1990) compared the learning of students who were trained in laundry skills with either single case (using one example) or general case (using multiple examples) simulation and natural settings. Students who were taught with a range of examples using general case programming in either the simulated or natural settings were able to generalize to untrained machines better than the students training with a single case were. Instruction, therefore, should be designed to provide multiple experiences or examples of the natural envi- 
ronment whether in the actual setting or with a simulation of that setting.

To illustrate, components from general case programming as suggested by Horner, Albin, and Ralph (1986) and Nietupski, Hamre-Nietupski, Clancy, and Veerhusen (1986) still provide accurate guidance for today's teachers. Their recommended steps include (a) defining the instructional universe, (b) determining the range of stimulus and response variations facing the students, (c) systematically varying simulations to provide a sufficient range of training exemplars, (d) sequencing and teaching the examples, (e) using community performance data to modify the simulations, (f) using simulations to provide intensified practice in problem areas, (g) testing with non-trained probe examples, and $(h)$ conducting training in one natural training session combined with in-class simulation training. Applying these techniques, teachers may choose a range of examples to be taught in both the simulated and the natural setting. Simulations can be used to provide extended intense practice; additionally, training is conducted in at least one natural setting.

In summary, the research on simulation training for community skills such as street crossing (Page, Iwata, \& Neef, 1976), bus riding (Neef, Iwata, $\&$ Page, 1978), vending machine operation (Browder, Snell, \& Wildonger, 1988), restaurant use (McDonnell \& Ferguson, 1988; Nietupski, Clancy, Wehrmacher, \& Parmer, 1985; van Den Pol, Iwata, Ivancic, Page, Neef, \& Whitley, 1981), and laundromat use (Morrow \& Bates, 1987) points to several recommendations for instruction and the development of any simulation training program. First, training conducted in several community locations can produce generalized responding. Second, simulation training can also produce generalized responding by incorporating a minimal amount of training in the natural environment. Third, testing for the generalization of the skills must occur in several additional novel locations. Finally, effective simulations must closely resemble the natural environment in appearance and in the behavioral response. Incorporating these recommendations into anchored instruction can assist teachers in creating simulations that more adequately depict real-life activities.

\section{Anchored Instruction}

Situated cognition has received considerable attention in the literature over the past decade. Brown, Collins, and Duguid (1989) and Lave (1988) believe that learning should be cognitively situated to take place in realistic settings under the guidance of "experts" who assist learners with the knowledge they need to solve problems and provide the cultural indoctrination necessary to be successful in certain community environments. A technology-based application of situated cognition has been termed "anchored instruction" (CTGV, 1990, 1993b). This application represents the use of 
visually rich video-based instructional contexts that emphasize learning through the application of knowledge and concepts to problems encountered in realistic environments. This approach contrasts with other methods of learning where knowledge and meaning are acquired through abstract activities to be stored in memory for later retrieval, such as memorizing community-relevant words out of context (Young, 1993).

When students with developmental disabilities are faced with socially related problems in work settings, they often have problems choosing the most appropriate response. Using anchored instruction, these students could be presented with short computer-based scenarios that provide video models of how students with similar characteristics handle such problems. Because of its link to computer-based systems (as opposed to videotaped examples), anchored instruction has several advantages. Initially, computer-based anchored instruction can provide learners with large numbers of video examples linked to specific problems and solutions. The learner can access these examples almost instantaneously, thus allowing students a closed link between the instruction and the video anchor.

Researchers and instructional developers continue to demonstrate that technology can provide one type of situated learning by using video that is "anchored" to academic (e.g., math, science) concepts, providing learners with examples of how experts use knowledge as tools to solve problems (CTGV, 1990, 1993a). Students and the teachers who facilitate their learning, explore video environments rich in information and examples that are used to solve increasingly complex problems (CTGV, 1993a; Young, 1993). The research supporting the effectiveness of situated learning in general (Griffin, 1995), and anchored instruction specifically (CTGV, 1993a, 1994), is promising and provides the opportunity to apply this technology-based situated learning approach to other instructional problem areas (Hedberg \& Alexander, 1994). For students with developmental disabilities, the development of video-based multimedia simulations (i.e., anchored instruction) may enhance the possibilities for instruction in a way that provides a better bridge between classroom-based and community-based instruction, allowing for repeated practice in the classroom.

\section{Computer and Video-based Instruction}

There is no singular seminal article that addresses "computer-based" or "video-based instruction" related to independent living and vocational skills for students with developmental disabilities. A majority of the studies researching the effectiveness of technology in special education have been done with students with mild intellectual disabilities and seem to have focused on the effects of computer-assisted academically related instruction (see Okolo, Bahr, \& Rieth, 1993; and Woodward \& Rieth, 1997 for reviews 
in this area). In contrast, the use of technology with students with more moderate developmental disabilities has generally focused on the adaptation of devices to compensate for a disability condition (i.e., adaptive positioning equipment), use of assistive technology to assist the student to perform specific tasks (i.e., single switches, braille readers, or voice synthesis) that provide increased opportunities for independence (Church \& Glennen, 1992; Garner \& Campbell, 1987), or training of students to understand cause-and-effect relationships.

Computer-based instruction. The literature provides evidence that computer-based instruction has been effective with persons who have more significant developmental disabilities in five areas related to functional and independent living skills: (a) establishing cognitive concepts such as cause-andeffect behaviors (Iacono \& Miller, 1989; Robinson, 1986), (b) teaching functional academic skills such as sight words and arithmetic facts (Conners \& Detterman, 1987; Lally, 1981; McGregor \& Axelrod, 1988; Powers \& Ball, 1983; Thorkildsen, Allard, \& Reid, 1983), (c) developing motivational leisure skills (Duffy \& Nietupski, 1985; McGregor \& Axelrod, 1988; Powers \& Ball, 1983; Sedlack, Doyle, \& Schloss, 1982), (d) providing communication aids using assistive technology (Iacono \& Miller, 1989), and (e) preparing computer-related job skills (Esposito \& Campbell, 1987). Literature that demonstrates the effectiveness of computer-based instruction for teaching basic skills or for fostering leisure skills provides evidence that with additional technological advances (i.e., video anchors) the power of this instruction can increase.

Video-based instruction. In the historic literature, Stephens and Ludy (1975) pointed out the value of using video, in the form of motion picture or videotaped instruction, for teaching action concepts (e.g., running, walking, drinking, standing) to students with mild or moderate disabilities. In a comparison of motion picture training with slides or live demonstration, the group receiving motion picture training performed better than either of the other groups. Using motion picture or videotape for instruction was better than slide training because teachers showed the skills involving motion in a systematic manner and maintained the students attention.

Haring, Kennedy, Adams, and Pitts-Conway (1987) indicated that students with autism were able to acquire and generalize social skills for grocery shopping after watching videotapes showing the correct process. Using the videotapes, the teachers had the flexibility to stop the tape and discuss the skills with the students. The students were first taught shopping skills within the natural environment, and the videotape was used to model and reinforce their social behaviors, enhancing the teacher's ability to promote generalization of the responses. Although the students improved in their shopping skills with training in the natural environment. their use of appro- 
priate social responses for shopping only increased after watching the videotapes.

Morgan and Salzburg (1992) conducted two studies designed to isolate the effects of video-assisted training from those of modeling and practice. Three adults with severe disabilities were able to communicate with words or short phrases and verbally identified problem situations in work settings. Only one adult was able to generalize to the video probe and the work setting. Results indicated that only after the other two participants viewed the video and then actively rehearsed the situations were they able to generalize to the video probe and thereafter, the work setting.

Although these traditional uses of motion video proved to be effective with the students in the Haring et al. (1987) and Stephens and Ludy (1975) studies, the role of their students was essentially passive; they sat and watched. Morgan and Salzburg (1992) also indicated that the addition of active participation increased the participants' ability to generalize to the work setting. The dimension of direct participation was missing. Interactive video or multimedia is an instructional technology which combines computer-assisted instruction and some form of educational video so that the learner can participate directly with the activity.

\section{Interactive-Video Instruction}

Payne and Antonow (1982) developed two types of interactive video programs because they felt that observation of the physical responses of the students would assist in determining if, and at what rate, skill acquisition was taking place. The first program gave the students the same feedback regardless of the response, and the second program either advanced to the next segment or repeated the segment contingent upon the response. Preliminary use of this program indicated that the students attended to the video and made an effort to complete the exercises.

By modifying these preliminary programs—but using video equipment similar to that described by Payne and Antonow (1982), Crusco et al. (1986) extended the work to determine the skills necessary for students with moderate to severe disabilities to use interactive video independently. The students were observed for sitting in seat, maintaining eye contact, responding to verbal prompts, pushing the correct button, and avoiding incorrect, inappropriate, or excessive button pushing. Only two of the twenty students were able to learn all the steps, indicating that the medium did not appear to be appropriate as an independent learning station for students with moderate or severe intellectual disabilities. These results indicate support for the combination of computer-based anchored instruction and community-based activities. Additional research that demonstrates the link between the two approaches is needed. 
In another example of the use of interactive videotape, Browning and White (1986) taught social skills to high school students with mild or moderate intellectual disabilities. The social skills taught were generic in nature (e.g., ways of solving problems in a variety of community and job situations). A field study conducted using these materials (Browning, White, Nave, \& Zembrosky-Barkin, 1986) with students with mild and moderate disabilities indicated gains in the students' knowledge and application of the curriculum skills. All students including those with moderate disabilities improved from pre- to post-test, indicating that the program was appropriate for high school students with this type of disability.

The Interactive Laserdisc for Special Education Technology program (Thorkildsen et al., 1983) developed and tested seven instructional interactive laserdisc programs for students with developmental disabilities. One program, "Matching and Prepositions," designed as individual learning stations for students with moderate to severe disabilities, could not be used by the students independently. Findings provide additional evidence that technologybased instruction is most effective when combined with other instruction.

Wissick, Lloyd, and Kinzie (1992) studied the effects of an interactive laserdisc-based grocery store simulation with three students with mild to moderate intellectual disabilities. Using a multiple-baseline-across-subjects design, students were assessed on the number of actions to locate and purchase an item for snack. All three students decreased the number of actions or steps needed to locate items in both the simulated and natural situations.

Similarly, Langone, Shade, Clees, and Day (in press) used a multipleprobe-across- subjects design to evaluate the effectiveness of a multimedia computer-based instructional program using photographs of target stimuli (i.e., cereal boxes as they appear on grocery store shelves) in an attempt to increase the likelihood that selection of specified cereal boxes would generalize to the grocery stores in the community. Students with moderate to severe disabilities were evaluated in both the simulated and the natural settings to measure the generalization of the discriminations. Results indicated that the mean and median duration to find target cereals decreased, coupled with an increase in the number of cereals found.

The literature indicates that there is promise for the use of technology; however, more research will be required to determine the exact relationship between these technology solutions and the actual generalization of the target skills in community environments. The use of the technology must replicate the community situation to enhance the learning environment for students and teachers. In this way, realistic exemplars can provide the teachers with a flexible and adaptable tool, and afford students increased opportunities for direct participation. Moreover, there is growing evidence that instructional applications of technology that provide video- 
based or multimedia simulations to teach functional living skills also provide repeated practice for students with developmental disabilities (Langone et. al., in press; Wissick et al., 1992).

\section{IMPLiCATIONS FOR DESIGNING Multimedia Simulations}

The effectiveness of multimedia instruction for teaching functional skills has been demonstrated in a number of studies (Alcantara, 1994; Haring et al., 1987; Haring, Breen, Weiner, Kennedy, \& Bednersh, 1995; McDonnell et al., 1984; Wissick et al., 1992). Much of the literature suggests that simulation training is most effective when paired with in vivo training (McDonnell \& Horner, 1985; McDonnell, Horner, \& Williams, 1984). Some research indicates that computer-based simulations alone may produce generalization and therefore be at least as effective as in vivo training in establishing skills in community-based settings, and potentially more cost effective (Langone et al., in press). This places "the onus on teachers...to provide environmental arrangements that establish discriminative stimuli associated with normative behaviors and reinforcers..." (Clees, 1995, p. 125).

\section{Design of Video-based Simulations}

The primary goal in creating a multimedia simulation is to enhance the learning environment. Such a simulation combines the capabilities of the computer and the video to present realistic representations of the environment, to provide appropriate feedback depending on the response, and to enable the teacher to review difficult material or steps in responding. Evaluation of the simulation's effectiveness is measured by the student's skills in the community or vocational situation. Current technology provides the teacher with tools to create situation- appropriate simulations.

As with the development of any instructional program for students with disabilities, a teacher first targets the functional adult living skills that the student needs to develop. These skills have been categorized into four domains: vocational, leisure/recreational, domestic living, and community living (Snell \& Grigg, 1987). In addition, an evaluation of the student's school, home, and community environments assists the teacher in developing appropriate functional goals.

To design video simulations for the identified skills, teachers can employ the steps from general case programming. Table 1 provides an example of general case programming applied to multimedia simulation development. Using the general case model, teachers can create a checklist of skills and develop an instructional design for the multimedia simulation video anchors. After creating a checklist of skills for the design, the instructional delivery (including the technology tools to develop the simulation and the 
Table 1

Designing Video-based Simulations Using the General Case Model

\begin{tabular}{lll}
\hline Steps for General Case & Description & Example \\
\hline 1. Define the & Define all the areas in which & Buying items for a bag \\
instructional domains. & the students may encounter & lunch at a grocery store.
\end{tabular}

2. Determine the range of stimulus and response variations facing the students.

\section{Systematically vary} simulations to provide a sufficient range of training exemplars.

\section{Sequence and teach the examples.}

\section{Use community} performance data to modify the simulations.

6. Use simulations to provide intensified practice in problem areas.

7. Test with nontrained probe examples.

8. Conduct training in one natural training session combined with in-class simulation training. the skill.

Determine all items or skills that the students would need to be able to perform.

Take photos or video of all situations that the student might encounter the items for snack and vary the teaching of each situation.

Structure the introduction of photos or video from gross discriminations to finer discriminations of examples.

If students have problems in a certain area in the community, retake photos.

Have students experience small amounts of time with the simulation each day.

Take additional photos and video to be used for testing students.

Depending on the severity of the disability and the strengths of each student, schedule appropriate visits to the community for training.
Students could buy plastic bags, paper bags, bread, mustard, raisins, cookies, box drinks, peanut butter, jam, lunch meat, or fruit.

Take photos or video of store isles in a variety of grocery stores.

Take close photos of items for students to discriminate between one or two examples. Take close-ups and wide angles in store aisles. Also take wide angle shots of grocery aisles.

If the students always select items on the bottom shelf. Take additional photos. Modify simulation to focus on all shelves.

If the students cannot discriminate between boxes of cookies. Provide additional discrimination practice with the simulation.

Take photos in a novel store or with additional items.

Take the students to the local grocery stores as needed. 
type of instructional setting for the simulation) needs to be considered.

\section{Instructional Delivery}

Past studies used videotape and videodisc technologies to present instructional scenarios and demonstrated that these scenarios did positively effect learned behaviors and potentially assisted learners in generalizing skills (e.g., Haring et al., 1987; Wissick et al., 1992). Unfortunately, videotape technology is linear in nature and requires considerable difficulty in locating and repeating important sequences on the tape that students may need to view for additional practice. Videodisc technology overcomes this weakness but requires considerable expense to create each videodisc, and the medium is limited by a relatively short number of video sequences that can be stored on one disc (i.e., 30 minutes of full-motion video).

Teachers need to know the both the limitations of the media and the potential of current technologies available to design simulations. If a video camcorder or a camera is used to take realistic photos in community or vocational settings, conversion to a digital format using a video capture board in a computer or a scanner is necessary. Fortunately, new digital cameras save photographs on a diskette and camcorders record in digital video format, providing easy portability to any multimedia program. Alternatively, teachers could have regular photographic film developed and indexed on a Kodak TM CD-Rom. They could then incorporate the photos using multimedia programs such as HyperStudio or PowerPoint (Microsoft, 1998).

Once developed, simulations that employ photographs and compressed video stored on a CD-ROM may allow for considerably more flexibility than previous technological solutions. This technology provides easy access to fullmotion or compressed video to (a) support instructional procedures designed to teach concepts, (b) highlight (increase the salience of) relevant dimensions of stimuli, and (c) allow for virtually unlimited practice. Alternatively, web-development tools are available to create interactivity using the World Wide Web. The advantage of publishing programs on the web is that other teachers in the area could access the simulations for their students. Future research efforts should address the effectiveness of current and emerging technologies (e.g., compressed video, DVD, World Wide Web) on improving generalization of skills to in vivo settings more efficiently.

Within the classroom, the teacher may choose to work with students individually on the simulation or have peer tutors work with students. The use of multimedia simulations can provide an environment for interaction with peers in an age-appropriate setting. Instead of replacing human contact, technology applications should provide age-appropriate instruction and increased interaction with peers and community members. Garner and Campbell (1987) listed three guidelines for effective use of technology with 
students with severe intellectual disabilities: (a) document changes in the behavior of the learner, (b) verify the functional relationship between the intervention and behavior of the learner, and (c) associate the outcomes with social and empirical validity; the latter is clearly an important factor when using simulations. Therefore, the degree to which teachers actively consider how technology (simulations in this case) can help students achieve personal goals, is also a highly relevant an issue.

\section{Considerations for Using Video-based Simulation}

The use of multimedia simulations remains an under-explored application of the technology that may effectively address numerous instructional and social domains for teaching students with developmental disabilities. Simulations can provide replications of lifelike situations and can be designed as an individual or group instructional tool. A variety of different simulations can be designed for students with developmental disabilities to assist the teacher in replicating aspects of community or vocational skills that require extra practice or are difficult to teach in the community. In addition, students would be using age-appropriate programs in conjunction with their peers. The technology would not be used to isolate the students but to create a learning environment shared with peers and teachers. When developing or selecting video-based simulations, teachers must also consider how social validity, design, and instructional delivery factors will interact to influence learning. Future research must also seek to identify the most effective combination of these factors that promotes efficient generalization.

A number of unanswered questions regarding the efficacy of interactive video still need to be examined. For example, an evaluation of the effects of video versus practice in the natural setting is needed to further our knowledge base. Also, future research questions should address the effectiveness of motion video versus still photographs.

Designers should have specific understanding of how effective models operate. Video-based simulations must include appropriate models (e.g., peers without disabilities) that actively participate in the task, providing the learner with guidance, support, and mediation in the learning situation. Models should pose additional/novel questions and give relevant feedback to provide added social validity and mediation to make the simulation even more authentic or conducive to learning. In many respects, the function attributed to the role model is directly in line with a philosophy inherent to the recently revised AAMR's definition of mental retardation (American Association on Mental Retardation, 1992) - that we must conceptualize the abilities of persons with developmental disabilities to include the degree and frequency to which they receive support from friends who are part of their daily lives. 
Teachers should use simulations with a specific understanding that both generalization and maintenance of community living skills are important. Simulations should be used to augment instruction that will take place in the real word. There is no reason to believe that teaching community living skills via simulation will automatically transfer to the natural environment. We also should consider the merit of using simulations to help maintain important skills that may decline if not practiced. Even if students have been able to demonstrate acquisition of a skill in the natural environment but do not have frequent opportunities to perform the skill, their skill level might deteriorate. Simulations may also help instruction to be more efficient by decreasing the number of community trials necessary for mastery.

We propose using the authenticity and anchored features of multimedia simulations to assist the learner in becoming as independent as possible within the simulation/classroom environment. In turn, the teacher works with the student to apply his or her learned skills in ways that promote smoother and more reliable transition to the real world.

\section{REFERENCES}

Alcantara, P. R. (1994). Effects of videotape instructional package on purchasing skills of children with autism. Exceptional Children, 61, 40-55.

American Association on Mental Retardation. (1992). Mental Retardation: Definition, Classification, and Systems of Supports (9th ed.). Washington, D.C.: AAMR.

Browder, D. M., Snell, M. E., \& Wildonger, B. A. (1988). Simulation and community-based instruction of vending machines with time delay. Education and Training in Mental Retardation, 23, 175-185.

Brown, J. S., Collins, A., \& Duguid, P. (1989). Situated cognition and the culture of learning. Educational Researcher, 18, 32-41.

Browning, P., \& White, W. A. T. (1986). Teaching life enhancement skills with interactive video-based curricula. Education and Training of the Mentally Retarded, 21, 236-244.

Browning, P., White, W. A. T., Nave, G., \& Zembrosky-Barkin, P. (1986). Interactive video in the classroom: A field study. Education and Training of the Mentally Retarded, 21, 85-92.

Church, G., \& Glennen, S. (1992). The handbook of assistive technology. San Diego, CA: Singular.

Clees, T. J. (1995). Self-recording of student's daily schedules of teachers' expectancies: Perspectives on reactivity, stimulus control, and generalization. Exceptionality, 5, 113-129.

Cognition and Technology Group at Vanderbilt. (1990). Anchored instruction and its relationship to situated cognition. Educational Researcher, 19 (6), 2-10.

Cognition and Technology Group at Vanderbilt. (1993a). Anchored instruction and situated cognition revisited. Educational Technology, 33 (3), 52-70.

Cognition and Technology Group at Vanderbilt. (1993b). Integrated media: Toward a theoretical framework for utilizing their potential. Journal of Special Education Technology, 12, $71-85$.

Cognition and Technology Group at Vanderbilt. (1994). Multimedia environments for developing literacy in at-risk students. In B. Means (ed.), Technology and education reform: The reality behind the promise. San Francisco: Jossey-Bass Publishers (p.23-56).

Conners, F. A., \& Detterman, D. K (1987). Information-processing correlates of computerassisted word learning by mentally retarded students. American Journal of Mental Deficiency, 91, 606-612. 
Crusco, A. H., Carter, P., McGrath, M., Payne, E., Antonow, J., \& O`Dell, S. (1986). Skill requirements for interactive video instruction with persons with mental retardation. Mental Retardation, 24, 99-105.

Cuvo, J., \& Klatt, K. (1992). Effects of community-based, videotape, and flashcard instruction of community referenced sight words on students with mental retardation. Journal of Applied Behavior Analysis, 25, 499-512.

Duffy, A. T., \& Nietupski, J. (1985). Acquisition and maintenance of video game initiation, sustaining and termination skills. Education and Training of the Mentally Retarded, 20, 157162.

Esposito, L., \& Campbell, P. H. (1987). Computers and severely and physically handicapped individuals. In J. Lindsey (Ed.), Computers and exceptional individuals (pp. 105-124). Columbus, $\mathrm{OH}$ : Merrill Publishing.

Garner, J. B., \& Campbell, P. H. (1987). Technology for persons with severe intellectual disabilities: Practical and ethical considerations. The Journal of Special Education, 21, 122-132.

Griffin, M. M. (1995). You can't get there from here: Situated learning, transfer, and map skills. Contemporary Educational Psychology, 20, 65-87.

Haring, T. G., Breen, C. G., Weiner, J., Kennedy, C. H., \& Bednersh, F. (1995). Using videotape modeling to facilitate generalized purchasing skills. Journal of Behavioral Education, $5,29-53$.

Haring, T. G., Kennedy, C. H., Adams, M. J., \& Pitts-Conway, V. (1987). Teaching generalization of purchasing skills across community settings to autistic youth using videotape modeling. Journal of Applied Behavior Analysis, 20, 89-96.

Hedberg, J., \& Alexander, S. (1994). Virtual reality in education: Defining researchable issues. Educational Media International, 31, 214220.

Horner, R. H., Albin, R. W., \& Ralph, G. (1986). Generalization with precision: The role of negative teaching examples in the instruction of generalized grocery item selection. Journal of the Association for Persons with Severe Handicaps, 11, 300-08.

Horner, R. H., McDonnell, J. J., \& Bellamy, G. T. (1986). Teaching generalized skills: General case instruction in simulation and community settings. In R. H. Horner, L. H. Meyer, \& H. D. Fredericks (Eds.), Education of learners with severe handicaps: Exemplary service strategies (pp. 289-314). Baltimore: Paul H. Brookes Publishing Co.

Iacono, T. A., \& Miller, J. F. (1989). Can microcomputers be used to teach communication skills to students with mental retardation? Education and Training of the Mentally Retarded, $24,32-44$.

Lally, M. (1981). Computer-assisted teaching of sight-word recognition for mentally retarded school children. American Journal of Mental Deficiency, 85, 383-388.

Langone, J. (1990). Teaching students with mild and moderate learning problems. Needham Heights, MA: Allyn and Bacon.

Langone, J., Shade, J., Clees, T., and Day, T. (In press). Effects of multimedia instruction on teaching functional discrimination skills to students with moderate/severe intellectual disabilities. International Journal of Disability, Development and Education.

Lave, J. (1988). Cognition in practice: Mind, mathematics and culture in everyday life. Cambridge, MA: Cambridge University Press.

Lewis, R. B. (1993). Special education technology classroom applications. Pacific Grove, CA: Brooks/Cole Publishing Company.

Lindsey, J. (1999). Computers and individuals with disabilities. Austin, TX: ProEd.

Male, M. (1997). Technology for Inclusion: Meeting the Special Needs of All Students (3rd ed.). Needham Heights, MA: Allyn and Bacon.

McDonnell, J. J., \& Ferguson, B. (1988). A comparison of general case in vivo and general case simulation plus in vivo training. The Journal of the Association for Persons with Severe Handicaps, 13, 116-124.

McDonnell, J., Hardman, M. L., Hightower, J., Keifer-O'Donnell, R., \& Drew, C. (1993). Impact of community-based instruction on the development of adaptive behavior of secondary-level students with mental retardation. American Journal on Mental Retardation, 97, 575-84. 
McDonnell, J. J., \& Horner, R. H. (1985). Effects of in vivo versus simulation-plus-in vivo training on the acquisition and generalization of grocery item selection by high school students with severe handicaps. Analysis and Intervention in Developmental Disabilities, 5, 323-343.

McDonnell, J. J., Horner, R. H., \& Williams, J. A. (1984). Comparison of three strategies for teaching generalized grocery purchasing to high school students with severe handicaps. The Journal of the Association for Persons with Severe Handicaps, 9, 123-133.

McGregor, G., \& Axelrod, S. (1988). Microcomputers in the classroom: Teaching students with severe handicaps to use a computer. Education and Treatment of Children, 11, 230-238.

Microsoft. (1998). PowerPoint [CD-ROM]. Redmond, WA: Microsoft Corporation.

Morgan, R. L., \& Salzburg, C. L. (1992). Effects of video-assisted training on employmentrelated social skills of adults with severe mental retardation. Journal of Applied Behavior Analysis, 25, 365-383.

Morrow, S. A., \& Bates, P. E. (1987). The effectiveness of three sets of school-based instructional materials and community training on the acquisition and generalization of community laundry skills by students with severe handicaps. Research in Developmental Disabilities, 8, 113-136.

Neef, N. A., Iwata, B. A., \& Page, T. J. (1978). Public transportation training: In vivo versus classroom instruction. Journal of Applied Behavior Analysis, 11, 331-344.

Neef, N. A., Lensbower, J., Hockersmith, I., DePalma, V., \& Gray, K. (1990). In vivo versus simulation training: An interactional analysis of range and type of training exemplars. Journal of Applied Behavior Analysis, 23, 447-458.

Nietupski, J., Clancy, P., Wehrmacher, L., \& Parmer, C. (1985). Effects of minimal versus length delay between simulated and in vivo instruction on community performance. Education and Training of the Mentally Retarded, 20, 190-195.

Nietupski, J., Hamre-Nietupski, S., Clancy, P., \& Veerhusen, K. (1986). Guidelines for making simulation an effective adjunct to in vivo community instruction. Journal of the Association for Persons with Severe Handicaps, 11, 12-18.

Okolo, C.M., Bahr, C.M., \& Rieth, H.J. (1993). A retrospective view of computer-based instruction. Journal of Special Education Technology, 12, 1-27.

Page, T. J., Iwata, B. A., \& Neef, N. A. (1976). Teaching pedestrian skills to retarded persons: Generalization from the classroom to the natural environment. Journal of Applied Behavior Analysis, 9, 433-444.

Payne, E., \& Antonow, J. (1982). Development and applications of user produced interactive videotape instructional materials. Journal of Special Education Technology, 5 (4), 33-36.

Powers, J., \& Ball, T. S. (1983). Video games to augment leisure programming in a state hospital residence for developmentally disabled clients. Journal of Special Education Technology, 6 (1), 48-57.

Robinson, L. (1986). Designing computer intervention for very young handicapped children. Journal of the Division for Early Childhood, 10, 209-215.

Sedlack, R. A., Doyle, M., \& Schloss, P. (1982). Video games: A training and generalization demonstration with severely retarded adolescents. Education and Training of the Mentally Retarded, 17, 332-336.

Snell, M. E., \& Browder, D. M. (1986). Community-referenced instruction: Research and issues. The Journal of the Association for Persons with Severe Handicaps, 11, 1-11.

Snell. M.E., \& and Grigg, N.C. (1987). Instructional assessment and curriculum development. In M.E. Snell (Ed.), Systematic Instruction of Persons with Severe Handicaps (3rd ed.) (pp. 64-109). New York: Merrill/Macmillan.

Stephens, W. E., \& Ludy, I. E. (1975). Action-concept learning in retarded children using photographic slides, motion picture sequences, and live demonstrations. American Journal of Mental Deficiency, 80, 277-280.

Stokes, T. F., \& Osnes, P. G. (1989). An operant pursuit of generalization. Behavior Therapy, $20,337-355$.

Thorkildsen, R., Allard, K., \& Reid, B. (1983). The interactive videodisc for special education project: Providing CAI for the mentally retarded. The Computing Teacher, 10(8), 73-76. 
van Den Pol, R. A., Iwata, B. A., Ivancic, M. T., Page, T. J., Neef, N. A., \& Whitley, F. P. (1981). Teaching the handicapped to eat in public places: Acquisition, generalization and maintenance of restaurant skills. Journal of Applied Behavior Analysis, 14, $61-69$.

Wagner, R. (1998). HyperStudio [CD-ROM]. El Cajon, CA: Roger Wagner Publishing.

Wissick, C.A., Lloyd, J.W., \& Kinzie, M.B. (1992). The effects of community training using a videodisc-based simulation. Journal of Special Education Technology, 11, 207-22.

Wolfe, P. S. (1994). Judgment of the social validity of instructional strategies used in community-based instructional sites. JASH, 19, 43-51.

Woodward, J., \& Rieth, H. (1997). A historical review of technology research in special education. Review of Educational Research, 67, 503-536.

Young, M. F. (1993). Instructional design for situated learning. Educational Technology Research and Development, 41, 43-58.

\section{AUTHOR INFORMATION}

Cheryl A. Wissick is an associate professor in the College of Education at the University of South Carolina. J. Emmett Gardner is an associate professor in the Department of Educational Psychology at The University of Oklahoma. John Langone is an associate professor at The University of Georgia.

\section{CONTACT INFORMATION}

Dr. Cheryl A. Wissick, College of Education, 235-H Wardlaw, The University of South Carolina, Columbia, SC 29208; email: cwissick@sc.edu. 